\title{
The National Institute for Health Research Hyperacute Stroke Research Centres and the ENCHANTED trial: the impact of enhanced research infrastructure on trial metrics and patient outcomes
}

Thompson G. Robinson ${ }^{1,2,11^{*}}$ (D), Xia Wang ${ }^{3}$, Alice C. Durham ${ }^{1}$, Gary A. Ford ${ }^{4}$, Joy Liao ${ }^{5}$, Sine Littlewood ${ }^{6}$, Christine Roffe ${ }^{7}$, Philip White ${ }^{8}$, John Chalmers ${ }^{3}$, Craig S. Anderson ${ }^{3,9,10}$ and on behalf of the ENCHANTED Investigators

\begin{abstract}
Background: The English National Institute for Health Research Clinical Research Network first established Hyperacute Stroke Research Centres (HSRCs) in 2010 to support multicentre hyperacute $(<9 \mathrm{~h})$ and complex stroke research. We assessed the impact of this investment on research performance and patient outcomes in a post-hoc analysis of country-specific data from a large multicentre clinical trial.

Methods: Comparisons of baseline, outcome and trial metric data were made for participants recruited to the alteplase-dose arm of the international Enhanced Control of Hypertension and Thrombolysis Stroke study (ENCHANTED) at National Institute for Health Research Clinical Research Network HSRCs and non-HSRCs between June 2012 and October 2015.

Results: Among 774 ENCHANTED United Kingdom participants (41\% female; mean age 72 years), 502 (64.9\%) were recruited from nine HSRCs and 272 (35.1\%) from 24 non-HSRCs. HSRCs had higher monthly recruitment rates (median 1.5, interquartile interval $1.4-2.2$ vs. $0.7,0.5-1.3 ; p=0.01$ ) and shorter randomisation-to-treatment times (2.6 vs. $3.1 \mathrm{~min} ; p=0.01$ ) compared to non-HSRCs. HSRC participants were younger and had milder stroke severity, but clinically important betweengroup differences in 90-day death or disability outcomes remained after adjustment for minimisation criteria and important baseline variables at randomisation, whether defined by ordinal modified Rankin scale score shift (adjusted OR 0.82 , 95\% Cl 0.62-1.08; $p=0.15$ ), scores 2 to 6 (adjusted OR 0.71, 95\% Cl 0.50-1.01; $p=0.05$ ), or scores 3 to 6 (adjusted $\mathrm{OR} 0.82,95 \% \mathrm{Cl} 0.57-1.17 ; p=0.27$ ). There was no significant difference in symptomatic intracerebral haemorrhage, nor heterogeneity in the comparative treatment effects between low- and standard-dose alteplase by HSRCS or non-HSRCs.
\end{abstract}

Conclusions: Infrastructure investment in HSRCs was associated with improved research performance metrics, particularly recruitment and time to treatment with clinically important, though not statistically significant, improvements in patient outcomes.

(Continued on next page)

\footnotetext{
* Correspondence: tgr2@le.ac.uk

${ }^{1}$ Department of Cardiovascular Sciences, University of Leicester, Leicester, United Kingdom

${ }^{2} \mathrm{NIHR}$ Leicester Biomedical Research Centre, Leicester, United Kingdom

Full list of author information is available at the end of the article
}

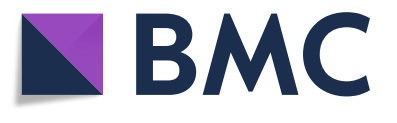

(c) The Author(s). 2019 Open Access This article is distributed under the terms of the Creative Commons Attribution 4.0 International License (http://creativecommons.org/licenses/by/4.0/), which permits unrestricted use, distribution, and reproduction in any medium, provided you give appropriate credit to the original author(s) and the source, provide a link to the Creative Commons license, and indicate if changes were made. The Creative Commons Public Domain Dedication waiver (http://creativecommons.org/publicdomain/zero/1.0/) applies to the data made available in this article, unless otherwise stated. 
(Continued from previous page)

Trial Registration: Unique identifier: NCT01422616.

Keywords: Acute ischaemic stroke, alteplase, clinical trials, symptomatic intracerebral haemorrhage, thrombolysis

\section{Background}

Intravenous (iv) alteplase (recombinant tissue plasminogen activator) is the approved medical reperfusion treatment for patients with acute ischaemic stroke and the earlier the treatment is given, the greater the benefit [1]. However, concerns over risks of intracranial haemorrhage have led to lower doses of alteplase being used in many Asian countries [2] after a lower dose $(0.6 \mathrm{mg} / \mathrm{kg})$ was approved in Japan. The Enhanced Control of Hypertension and Thrombolysis Stroke Study (ENCHANTED) assessed a low dose $(0.6 \mathrm{mg} / \mathrm{kg}$ body weight) compared to the standard dose $(0.9 \mathrm{mg} / \mathrm{kg})$ of iv alteplase in acute ischaemic stroke patients fulfilling standard criteria for thrombolysis ('clot-busting' therapy) in improving treatment efficacy through reduced 90-day death and disability, and safety through reduced symptomatic intracerebral haemorrhage (bleeding associated with neurological deterioration or death) [3]. The primary outcome of the study was to demonstrate non-inferiority of low- compared to standard-dose alteplase on 90-day death and disability, defined by scores 2 to 6 on the modified Rankin scale $(\mathrm{mRS})$. The $\mathrm{mRS}$ is a global seven-level assessment of disability, where scores of 0 to 1 indicate a favourable outcome with/without symptoms but no disability; 2 to 5 indicate increasing levels of disability and dependency, requiring no (2), weekly (3), daily (4), and 24-h (5) physical help; and 6 indicates death. Whilst low-dose alteplase was not non-inferior to the standard dose, it was clearly non-inferior on a secondary efficacy outcome of shift ('improvement') in measures of daily function according to the full range of scores on the mRS scores, and reduced the risk of symptomatic intracerebral haemorrhage [3].

The United Kingdom had the second highest patient recruitment $(n=774,23.3 \%)$ to the ENCHANTED alteplase dose-arm after China, supported by the National Institute for Health Research (NIHR) Clinical Research Network infrastructure for clinical research through the National Health Service (NHS). In particular, NIHR has made significant investment in eight (expanding to 10 during the conduct of ENCHANTED) Hyperacute Stroke Research Centres (HSRCs), established in 2010 with an initial investment of $£ 3.66$ million over 3 years. These high patient volume centres ( $>1000$ stroke admissions per annum) provide multi-disciplinary expertise in hyperacute stroke clinical management (including engagement of pre-hospital, emergency department, interventional neuroradiology, neuroradiology, neuro-critical care, and neurosurgical staff), 24/7 availability of advanced neuroimaging, 7-day per week extended hours (07:00 to 22:00, minimum) resident research staffing, and a track record of performing against specialty performance objectives (in particular recruitment to the hyperacute $(<9 \mathrm{~h}$ of stroke onset) and complex trial portfolio (a trial requiring complex diagnostics, or surgery/complex intervention, or phase 1 or 2 commercial study)). In addition, the HSRC selection process includes a review of recent scores on the Sentinel Stroke National Audit Programme, particularly related to hyperacute process metrics, including door-to-scan time, rates of admission to Hyperacute Stroke Units within $4 \mathrm{~h}$ and thrombolysis rates, where scores at the highest level (A or B) should be consistently achieved. HSRCs are subject to annual review by an independent panel to maintain their accreditation. The return on such investment in infrastructure might extend beyond improved research performance; therefore, whilst we primarily evaluated the impact of HSRCs on trial metrics, we also assessed the effect on patient outcomes in NIHR Clinical Research Network sites participating in ENCHANTED in England.

\section{Methods}

Design

The ENCHANTED trial was an international, multi-centre, prospective, randomised, open-label, blinded-endpoint trial with a $2 \times 2$ partial-factorial design to assess the effectiveness of low- versus standard-dose alteplase (completed arm) and more intensive versus guideline-recommended control of blood pressure (ongoing arm), the details of which are outlined in detail elsewhere [3, 4]. In brief, patients admitted to hospital with a clinical diagnosis of acute ischaemic stroke confirmed on brain imaging and fulfilling local criteria for thrombolysis treatment administered within $4.5 \mathrm{~h}$ of symptom onset, were randomly assigned to the respective dose arm between 18 June 2012 and 14 October 2015. Randomised patients received low-dose (0.6 $\mathrm{mg} / \mathrm{kg}$; $15 \%$ as bolus, $85 \%$ as infusion over $1 \mathrm{~h}$; maximum dose $60 \mathrm{mg}$ ) or standard-dose $(0.9 \mathrm{mg} / \mathrm{kg} ; 10 \%$ as bolus, $90 \%$ as infusion over $1 \mathrm{~h}$; maximum dose $90 \mathrm{mg}$ ) iv alteplase. Otherwise, all patients received active care and best practice management according to local guidelines. The study protocol was approved by the appropriate ethics committee at each participating centre, and written 
informed consent was obtained from the patient or an appropriate surrogate.

\section{Procedures}

Key demographic and clinical characteristics were recorded at the time of enrolment. Stroke severity was measured using the National Institutes of Health Stroke Scale at baseline, at $24 \mathrm{~h}$, and on day 7 (or earlier, upon discharge from hospital). Uncompressed digital images of all baseline and follow-up brain scans (CT, MRI and angiogram) were collected, with details of transfer, interpretation and intracranial haemorrhage definitions provided (Additional file 1).

As previously stated, the primary clinical outcome was the combined endpoint of death or disability at 90 days, defined by scores of 2 to 6 on the mRS. Other efficacy outcomes included an ordinal mRS shift and the combined endpoint of death or major disability (mRS scores of 3 to 6) at 90 days. The secondary (safety) outcome was symptomatic intracerebral haemorrhage, defined according to several criteria from other studies (Additional file 1). Additional new analyses in this manuscript focus on trial performance metrics, including total recruitment, monthly recruitment rate, and times to randomisation and treatment.

\section{Statistical analysis}

The association of HSRC recruitment on global functional outcome (analysis of the full range of day $90 \mathrm{mRS}$ scores) was estimated using ordinal logistic regression after the assumption of proportionality of the odds was confirmed from a likelihood ratio test. Adjustment was made for the ENCHANTED pre-specified minimisation variables and several baseline covariates at randomisation, and additionally for aspects of management over the first 7 days after hospital admission. In patients from HSRCs and non-HSRCs, the heterogeneity of the alteplase treatment effect was tested by adding an interaction term to the statistical models. Data are reported with odds ratios (ORs) and 95\% confidence intervals
(CIs). A two-sided $p$ value of less than 0.05 was considered statistically significant. SAS version 9.3 (SAS Institute, Cary, NC) was used for all analyses.

\section{Role of the funding source}

The sponsors had no role in the study design, data collection, data analysis, data interpretation or writing of the report. All authors had full access to the study data. The corresponding author had final responsibility for the decision to submit the paper for publication.

\section{Results}

These analyses included 774 patients (41\% female; mean age 72 years) randomised to the ENCHANTED trial in the United Kingdom (England 768, non-England 6), including 9 of the 10 established HSRCs (one site did not participate in ENCHANTED). Although HSRCs comprised only $27 \%$ (9/33) of recruiting sites, they contributed nearly two-thirds $(502,64.9 \%)$ of participants and had a significantly higher median monthly recruitment rate than non-HSRCs (1.5 (interquartile interval 1.4-2.2) vs. 0.7 $(0.5-1.3))$. With respect to performance on other trial metrics, Table 1 shows that HSRC patients had significantly shorter randomisation-to-treatment times, as well as differences in other aspects of management, including angiography and endovascular therapy. Table 2 shows that participants recruited from HSRCs were significantly younger, and had milder severity of stroke, as assessed by National Institutes of Health Stroke Scale or Glasgow coma score. HSRC patients were also significantly less likely to develop fever, receive antiplatelet therapy in the first $24 \mathrm{~h}$ after thrombolysis, or have rehabilitation in the first 7 days, but were more likely to receive subcutaneous heparin (Additional file 2: Table S1).

Compared to non-HSRC patients, HSRC-treated patients were significantly less likely to be dead or disabled at 90 days, whether defined by ordinal shift (unadjusted OR $0.68,95 \%$ CI $0.52-0.89 ; p=0.005$ ), scores 2 to 6 (unadjusted OR 0.63 , 95\% CI 0.46-0.86; $p<0.0001$ ), or scores 3 to 6 (unadjusted OR 0.69, 95\% CI 0.50-0.82;

Table 1 Selected trial metrics and management of patients by type of stroke research centre

\begin{tabular}{|c|c|c|c|c|}
\hline & $\begin{array}{l}\text { All United Kingdom } \\
(n=774)\end{array}$ & $\begin{array}{l}\text { HSRC } \\
(n=502)\end{array}$ & $\begin{array}{l}\text { Non-HSRC } \\
(n=272)\end{array}$ & $p$ value \\
\hline Number of recruiting centres $(n)$ & 33 & 9 & 24 & \\
\hline Monthly recruitment rate (median, IQR) & $1.0(0.6-1.5)$ & $1.5(1.4-2.2)$ & $0.7(0.5-1.3)$ & 0.01 \\
\hline Time from stroke onset to treatment, mins (median, IQR) & $139(110-175)$ & $135.5(110-178)$ & $140.0(111-174)$ & 0.65 \\
\hline Time from randomisation to treatment, mins (median, IQR) & $2.8(0.2-5.4)$ & $2.6(-0.6$ to 5.3$)$ & $3.1(1.1-5.7)$ & 0.01 \\
\hline Alteplase given $(n / N, \%)$ & 765/774 (98.8) & 498/502 (99.2) & $267 / 272(98.2)$ & 0.20 \\
\hline Cerebral angiogram $(n / N, \%)$ & $22 / 774(2.8)$ & 20/502 (4.0) & $2 / 272(0.7)$ & 0.009 \\
\hline Occluded vessel $(n / N, \%)$ & 18/22 (81.8) & $17 / 20(85.0)$ & $1 / 2(50.0)$ & 0.22 \\
\hline Endovascular therapy $(n / N, \%)$ & $16 / 22(72.7)$ & $15 / 20(75.0)$ & $1 / 2(50.0)$ & 0.45 \\
\hline
\end{tabular}

$H S R C$ hyperacute stroke research centre, IQR interquartile range 
Table 2 Baseline characteristics of patients by type of stroke research centre

\begin{tabular}{|c|c|c|c|c|}
\hline & $\begin{array}{l}\text { All United Kingdom } \\
(n=774)\end{array}$ & $\operatorname{HSRC}(n=502)$ & Non-HSRC $(n=272)$ & $p$ value \\
\hline Time from stroke onset to randomisation, hours (median, IQR) & $2.3(1.8-2.9)$ & $2.2(1.8-2.9)$ & $2.3(1.8-2.8)$ & 0.82 \\
\hline Age, years (mean, SD) & $72(14)$ & $71(14)$ & $75(13)$ & 0.0002 \\
\hline Female $(n / N, \%)$ & $318 / 774(41.1)$ & 203/502 (40.4) & $115 / 272(42.3)$ & 0.62 \\
\hline Non-Asian ethnicity $(n / N, \%)$ & 757/774 (97.8) & 490/502 (97.6) & $267 / 272(98.2)$ & 0.62 \\
\hline Systolic BP, mmHg (mean, SD) & $151(20)$ & $151(19)$ & $152(20)$ & 0.52 \\
\hline Heart rate, bpm (mean, SD) & $80(18)$ & $82(18)$ & $77(17)$ & 0.0003 \\
\hline NIHSS score (median, IQR) & $7(5-13)$ & $7(5-11)$ & $8(5-14)$ & 0.01 \\
\hline GCS score (median, IQR) & $15(14-15)$ & $15(14-15)$ & $15(14-15)$ & 0.01 \\
\hline History of hypertension $(n / N, \%)$ & $472 / 774(61.0)$ & 299/502 (59.6) & $173 / 272(63.6)$ & 0.27 \\
\hline History of coronary artery disease $(n / N, \%)$ & $112 / 774(14.5)$ & $73 / 502(14.5)$ & 39/272 (14.3) & 0.94 \\
\hline AF confirmed on ECG $(n / N, \%)$ & 188/772 (24.4) & $117 / 500(23.4)$ & $71 / 272(26.1)$ & 0.40 \\
\hline Hypercholesterolaemia $(n / N, \%)$ & 260/774 (33.6) & $161 / 502(32.1)$ & 99/272 (36.4) & 0.22 \\
\hline Current smoker $(n / N, \%)$ & $124 / 770(16.1)$ & $92 / 501(18.4)$ & $32 / 269(11.9)$ & 0.02 \\
\hline Pre-stroke symptoms on the mRS $(n / N, \%)$ & 240/773 (31.0) & 149/501 (29.7) & $91 / 272(33.5)$ & 0.29 \\
\hline Antihypertensive therapy $(n / N, \%)$ & 429/774 (55.4) & $264 / 502(52.6)$ & $165 / 272(60.7)$ & 0.03 \\
\hline Warfarin anticoagulation $(n / N, \%)$ & 23/773 (3.0) & 18/501 (3.6) & $5 / 272(1.8)$ & 0.17 \\
\hline Aspirin/other antiplatelet $(n / N, \%)$ & $273 / 773(35.3)$ & 178/501 (35.5) & 95/272 (34.9) & 0.87 \\
\hline Statin/other lipid lowering ( $n / N, \%)$ & 103/773 (13.3) & $66 / 501(13.2)$ & $37 / 272(13.6)$ & 0.87 \\
\hline \multicolumn{5}{|l|}{ Brain imaging features } \\
\hline$C T$ scan used $(n / N, \%)$ & 769/774 (99.4) & $502 / 502(100)$ & $267 / 272(98.2)$ & 0.002 \\
\hline MRI scan used $(n / N, \%)$ & $8 / 774(1.0)$ & $4 / 502(0.8)$ & $4 / 272(1.5)$ & 0.38 \\
\hline Visible early ischaemic changes $(n / N, \%)$ & 230/774 (29.7) & 156/502 (31.1) & $74 / 272(15.1)$ & 0.26 \\
\hline Visible cerebral infarction ( $n / N, \%)$ & 144/774 (18.6) & 103/502 (20.5) & $1 / 272(0.4)$ & 0.06 \\
\hline \multicolumn{5}{|l|}{ Final diagnosis } \\
\hline Non-stroke $(n / N, \%)$ & $50 / 768(6.5)$ & $33 / 498(6.6)$ & $17 / 270(6.3)$ & 0.86 \\
\hline Large artery occlusion (n/N, \%) & 170/713 (23.8) & $129 / 461(28.0)$ & $41 / 252(16.3)$ & 0.003 \\
\hline Small vessel disease $(n / N, \%)$ & 139/713 (19.5) & $91 / 461(19.7)$ & $48 / 252(19.0)$ & \\
\hline Cardioembolism (n/N, \%) & $181 / 713(25.4)$ & 106/461 (23.0) & 75/252 (29.8) & \\
\hline Other or uncertain aetiology $(n / N, \%)$ & 223/713 (31.3) & 135/461 (29.3) & $88 / 252(34.9)$ & \\
\hline
\end{tabular}

HSRC Hyperacute Stroke Research Centre, BP blood pressure, NIHSS National Institutes of Health Stroke Scale, GCS Glasgow coma scale, AF atrial fibrillation, $m R S$ modified Rankin scale

$p=0.017)$ on the mRS. There remained clinically important, though not statistically significant differences in mortality or disability outcomes at 90 days after adjustment for the minimisation criteria and baseline prognostic variables, whether defined by ordinal shift (adjusted OR 0.82 , 95\% CI $0.62-1.08 ; p=0.15$ ), scores 2 to 6 (adjusted OR $0.71,95 \%$ CI $0.50-1.01 ; p=0.05$ ), or scores 3 to 6 (adjusted OR $0.82,95 \%$ CI $0.57-1.17 ; p=0.27$ ). There were no significant differences after further adjustment for imbalances in early management (Table 3).

Moreover, no significant differences were seen in symptomatic intracerebral haemorrhage between HSRC and non-HSRC patient groups across a broad range of definitions (Additional file 2: Table S2), nor were there any significant differences in the treatment effect
(Additional file 2: Tables S3 and S4) or safety (Additional file 2: Table S5) between low- and standard-dose alteplase between HSRC and non-HSRC treated patients.

\section{Discussion}

In these country-specific secondary analyses of the ENCHANTED trial of low- versus standard-dose alteplase in thrombolysis-eligible acute ischaemic stroke patients, we have shown significantly higher recruitment rates and improved trial performance metrics in relation to local research infrastructure investment. HSRCs were established with additional funding to support hyperacute complex stroke trials, and while only 9 of the 33 United Kingdom recruiting centres (England 31, non-England 2) in ENCHANTED were HSRC designated, they recruited 
Table 3 Clinical outcomes at 90 days in patients by type of stroke research centre

\begin{tabular}{|c|c|c|c|c|c|c|c|}
\hline & $n(\%)$ & Unadjusted OR (95\% Cl) & $p$ value & Adjusted $\mathrm{OR}^{\mathrm{a}}(95 \% \mathrm{Cl})$ & $p$ value & Adjusted $\mathrm{OR}^{\mathrm{b}}(95 \% \mathrm{Cl})$ & $p$ trend \\
\hline \multicolumn{8}{|c|}{ Death or disability (mRS 2 to 6) } \\
\hline Non-HSRC & $165 / 256(64.5)$ & 1.0 & 0.004 & 1.0 & 0.05 & 1.0 & 0.87 \\
\hline HSRC & 249/467 (53.3) & $0.63(0.46-0.86)$ & & $0.71(0.50-1.01)$ & & $0.97(0.64-1.46)$ & \\
\hline \multicolumn{8}{|c|}{ Death or disability (mRS 3 to 6) } \\
\hline Non-HSRC & $121 / 256(47.3)$ & 1.0 & 0.017 & 1.0 & 0.27 & 1.0 & 0.82 \\
\hline HSRC & 178/467 (38.1) & $0.69(0.50-0.82)$ & & $0.82(0.57-1.17)$ & & $1.05(0.68-1.62)$ & \\
\hline \multicolumn{8}{|l|}{ Death } \\
\hline Non-HSRC & $34 / 272(12.5)$ & 1.0 & 0.24 & 1.0 & 0.60 & 1.0 & 0.58 \\
\hline HSRC & 49/502 (9.8) & $0.76(0.48-1.21)$ & & $0.87(0.51-1.47)$ & & $1.21(0.61-2.39)$ & \\
\hline \multicolumn{8}{|c|}{ mRS categories } \\
\hline Non-HSRC & & 1.0 & 0.005 & 1.0 & 0.15 & 1.0 & 0.77 \\
\hline 0 & $37(14.5)$ & & & & & & \\
\hline 1 & $54(21.1)$ & & & & & & \\
\hline 2 & $44(17.2)$ & & & & & & \\
\hline 3 & 45 (17.6) & & & & & & \\
\hline 4 & $23(9.0)$ & & & & & & \\
\hline 5 & $19(7.4)$ & & & & & & \\
\hline 6 & $34(13.3)$ & & & & & & \\
\hline HSRC & & $0.68(0.52-0.89)$ & & $0.82(0.62-1.08)$ & & $1.05(0.77-1.43)$ & \\
\hline 0 & $100(21.4)$ & & & & & & \\
\hline 1 & $118(25.3)$ & & & & & & \\
\hline 2 & $71(15.2)$ & & & & & & \\
\hline 3 & $61(13.1)$ & & & & & & \\
\hline 4 & $42(9.0)$ & & & & & & \\
\hline 5 & $26(5.6)$ & & & & & & \\
\hline 6 & 34 (13.3) & & & & & & \\
\hline
\end{tabular}

$\mathrm{Cl}$ confidence interval, HSRC hyperacute stroke research centre, $m R S$ modified Rankin score, OR odds ratio, aOR adjusted odds ratio

${ }^{a}$ Model 1: adjusted analysis for minimisation variables including National Institutes of Health Stroke Scale score and time from stroke onset to randomisation, and baseline variables: age, sex, ethnicity, systolic blood pressure, heart rate, hypercholesterolaemia, current smoker, premorbid mRS, premorbid use of antihypertensive therapy, aspirin or other antiplatelet agent, and randomised treatment (low dose versus standard dose)

${ }^{b}$ Model 2: as Model 1, plus systolic blood pressure at $24 \mathrm{~h}$, fever occurrence, nasogastric feeding given, subcutaneous heparin used, patient mobilised by therapist, any stroke unit admission, any neurosurgery performed, and any rehabilitation given

nearly two-thirds of all the patients in the United Kingdom, and with faster recruitment and shorter randomisation-to-treatment times. Furthermore, whilst 90\% of English HSRCs participated in ENCHANTED, the participation rate was far lower amongst the English non-HSRC sites, at 21\% (24/117). HSRC-treated patients had significantly better clinical outcomes in the unadjusted analyses, which remained clinically, though not quite statistically, significant after adjustment for minimisation criteria and differences in baseline characteristics. No differences were seen compared to non-HSRC-treated patients after adjustment for early management, which may reflect better care processes in HSRCs.

The importance of research is explicitly stated in The Handbook to the NHS Constitution in the United
Kingdom [5]. Indeed, the NIHR Clinical Research Network was specifically established with the aim of improving the health and well-being of the nation, through research focused on the needs of patients and the public. In the field of stroke, additional funding in the HSRC network was directed to deliver hyperacute (randomisation $<9 \mathrm{~h}$ of symptom onset) and/or complex studies (using advanced neuroimaging or other diagnostics, interventional neuroradiology or neurosurgery). An assessment of any positive impact of this research infrastructure investment on research performance measures and health outcomes would be important to demonstrate to NHS commissioners and providers the value of research infrastructure funding. To our knowledge, this is the first such evaluation on the return on this HSRC investment. 
Analyses of practice-based research networks in the United States have shown improved clinical outcomes in participating practices [6], whilst previous NIHR data analyses have shown that Trusts with the lowest mortality for adult non-elective admissions had higher levels of research patient recruitment and funding than those with expected or higher mortality rates [7]. The survival benefits for research participants and other patients separately persist after adjustment for staffing and other hospital structural factors such as medical and staffing ratios per bed, critical care versus general bed ratios, and affiliations with a university [7]. Our study provides further support for positive impact of research investment on research participation, trial performance metrics and patient recruitment.

In addition, though we were unable to clearly demonstrate a statistically significant association between research investment and improved patient outcomes in adjusted (as opposed to unadjusted) analyses, this is most likely related to the sample size and thus the limited statistical power to confirm modest but still clinically important differences. Indeed, even after adjustment for minimisation criteria and baseline differences, we were able to demonstrate a borderline statistical, but clinically significant, 29\% reduction in 90-day death and disability, defined by a mRS of 2 to 6 . Jonker and Fisher [8] were also unable to show that the degree of NIHR portfolio clinical research activity was significantly related to risk rating for overall performance by the Care Quality Commission. Nonetheless, another study of high-risk conditions, including stroke, has shown a significant correlation between academic output, as measured by citations per hospital admission, and overall mortality [9]. Furthermore, a study of colorectal cancer outcome by the NIHR Cancer Research Network reported a significant reduction in post-operative mortality and improved 5-year survival in research-active centres [10]. Indeed, there are other examples of improved patient outcome and adherence to guidelines in hospitals participating in related clinical trials compared to other hospitals, for example, in relation to the management of non-ST-segment elevation acute coronary syndromes [11].

Several possible mechanisms have been proposed for why clinical research may improve health outcomes, including enhanced infrastructure and organisation, multidisciplinary and institutional collaboration, education and training, specialisation, and care processes, particularly in the uptake and adherence to guideline-recommended care $[12,13]$. In particular, HSRCs provide enhanced infrastructure, including 7-day per week extended hours (07:00 to 22:00, minimum) resident research staffing and improved collaboration between key specialties in hyperacute stroke management. These mechanisms are supported by two recent systematic reviews, which concluded that a strong research culture has patient, staff and organisational benefits [14], and that institution and clinician engagement in clinical trials has greater adherence to guidelines and better outcomes [15]. Whilst we were unable to explore these themes retrospectively, a qualitative study has reinforced their relevance in an NIHR context at the NIHR Oxford Biomedical Research Centre [16]. Another potential explanation for the research and clinical benefits of HSRCs on patient care processes and outcomes may relate to a 'volume effect' [17]. One of the criteria included in HSRC designation is the number of annual stroke admissions, with the average annual admission rate in HSRCs being higher compared to non-HSRCs (1713 (range 820-2920) vs. 971 (288-1556)) [18].

We acknowledge that our study is limited by being based on data derived from a single country where the open-label trial design may have introduced various biases despite our efforts at concealment of treatment allocation, assessment of adverse events and blinded evaluation of clinical outcomes using established criteria. Moreover, as the ENCHANTED trial included patients with generally milder stroke severity with a slightly longer treatment delay from onset than in previous trials or registries [19], there may be further concerns over the generalisability of these data, and imprecision in the estimates of the effects may have arisen from variability in the assessment of the mRS [20]. In addition, the proportion of female patients is lower than might be expected given the age demographic of stroke, and highlights regional differences in sex distribution between registry and trial populations, with male predominance being previously reported in stroke thrombolysis trials [21]. Finally, it is difficult to determine if the improved research performance of HSRCs was related to the research infrastructure investment associated with their designation as an HSRC, or whether these were already high performing research centres or clinical Hyperacute Stroke Units. Only one centre changed status from a non-HSRC to a HSRC during the trial, with average monthly recruitment rates of 1.3 and 2.4 over 29 months before and 4.5 months after change in HSRC status, respectively. However, five HSRCs participated in the Third International Stroke Trial, between May 2000 and July 2011 [22], which is a similar trial comparing iv thrombolysis with control treatment up to $6 \mathrm{~h}$ following acute ischaemic stroke onset. Average monthly recruitment rates increased by approximately $50 \%$ from 0.8 and 1.1 between April 2007 and March 2010 (pre-HSRC status) and April 2010 to July 2011 (HSRC status), respectively.

\section{Conclusions}

We have demonstrated a positive impact of research infrastructure investment, with significant improvements in overall recruitment and other trial performance 
metrics in HSRCs, including monthly recruitment rates and time to randomisation and treatment. This also led to broader clinically important benefits in patient outcomes, even after adjustment for differences in minimisation criteria and baseline characteristics, though these did not reach statistical significance. This should provide confidence to commissioners and providers of the importance of research investment and participation, as highlighted in the NHS Constitution.

\section{Additional files}

Additional file 1: Imaging transfer and analysis, and definitions of symptomatic intracerebral haemorrhage. (DOCX $40 \mathrm{~kb}$ )

Additional file 2: Table S1. Use of alteplase and management details from randomisation to day 7 by hyperacute stroke research centres (HSRCs) and non-HSRCs. Table S2. Key secondary outcome of symptomatic intracerebral haemorrhage across all definitions by HSRCs and nonHSRCs. Table S3. Key efficacy outcomes by randomised treatment and HSRCs and non-HSRCs. Table S4. Key efficacy outcomes by randomised treatment and HSRCs and non-HSRCs. Table S5. Key safety outcome of symptomatic intracerebral haemorrhage by randomised treatment and HSRCs and non-HSRCs. (DOCX $49 \mathrm{~kb}$ )

\section{Abbreviations}

Cl: confidence interval; ENCHANTED: Enhanced Control of Hypertension and Thrombolysis Stroke Study; HSRC: Hyperacute Stroke Research Centre; iv: intravenous; mRS: modified Rankin scale; NHS: National Health Service; NIHR: National Institute for Health Research; OR: odds ratio

\section{Acknowledgements}

Not applicable.

\section{Funding}

The study is supported by grants from the National Health and Medical Research Council (NHMRC) of Australia, the Stroke Association of the United Kingdom, the Ministry of Health and the National Council for Scientific and Technological Development of Brazil (CNPQ: 467322/2014-7, 402388/20135), and the Ministry for Health, Welfare and Family Affairs of the Republic of Korea (HI14C1985)

\section{Availability of data and materials}

The datasets analysed during the current study are available from the corresponding author on reasonable request.

\section{Authors' contributions}

TGR drafted the manuscript for content; TGR and CSA contributed to acquisition of the data; TGR and CSA were responsible for the study concept and co-ordination; XW was responsible for statistical analyses; TGR, XW and CSA were responsible for interpretation of the data. All authors contributed to writing and editing of the manuscript, and approved the final version.

\section{Ethics approval and consent to participate}

The study protocol was approved by the appropriate ethics committee at each participating centre, and written informed consent was obtained from the patient or an appropriate surrogate.

\section{Consent for publication}

Not applicable.

\section{Competing interests}

TGR is an NIHR Senior Investigator; PW reports institutional research grants from Microvention Terumo, advisory panel fees from Stryker, and educational consulting fees from Microvention; JC reports research grants and lecture fees from Servier; CSA reports advisory panel fees from Amgen, speaking fees from Takeda China, research grants from Takeda China and NHMRC, and holds a Senior Principle Research Fellowship of the NHMRC.

\section{Publisher's Note}

Springer Nature remains neutral with regard to jurisdictional claims in published maps and institutional affiliations.

\section{Author details}

${ }^{1}$ Department of Cardiovascular Sciences, University of Leicester, Leicester, United Kingdom. ${ }^{2} \mathrm{NIHR}$ Leicester Biomedical Research Centre, Leicester, United Kingdom. ${ }^{3}$ The George Institute for Global Health, Faculty of Medicine, University of New South Wales, Sydney, NSW, Australia. ${ }^{4}$ Oxford University Hospitals NHS Foundation Trust and Radcliffe Department of Medicine, University of Oxford, Oxford, United Kingdom. ${ }^{5}$ NIHR Specialty Cluster A Co-ordinating Centre, Imperial College London, London, United Kingdom. ${ }^{6} \mathrm{NIHR}$ Clinical Research Network National Co-ordinating Centre, Leeds, United Kingdom. ${ }^{7}$ Stroke Research in Stoke Institute for Applied Clinical Studies, Keele University, Staffordshire, United Kingdom. ${ }^{8}$ Institute of Neuroscience Newcastle University and Newcastle upon Tyne Hospitals NHS Trust, Newcastle upon Tyne, United Kingdom. ${ }^{9}$ Neurology Department, Royal Prince Alfred Hospital, Sydney, NSW, Australia. ${ }^{10}$ The George Institute China at Peking University Health Sciences Center, Beijing, China. ${ }^{11} \mathrm{BHF}$ Cardiovascular Research Centre, Glenfield Hospital, Groby Road, Leicester LE3 9QP, United Kingdom

Received: 22 August 2018 Accepted: 20 January 2019

Published online: 13 February 2019

\section{References}

1. Emberson J, Lees KR, Lyden P, Blackwell L, Albers G, Bluhmki E, et al. Effect of treatment delay, age and stroke severity on the effects of intravenous thrombolysis with alteplase for acute ischaemic stroke: a meta-analysis of individual patient data from randomised trials. Lancet. 2014;384:1929-35.

2. Sharma VK, Ng KW, Venketasubramanian N, Saqqur M, Teoh HL, Kaul S, et al. Current status of intravenous thrombolysis for acute ischemic stroke in Asia. Int J Stroke. 2011;6:523-30

3. Anderson CS, Robinson T, Lindley Rl, Arima H, Lavados PM, Lee T-H, et al. Trial of low-dose versus standard-dose intravenous alteplase in patients with acute ischemic stroke. N Engl J Med. 2016;374:2313-23.

4. Huang Y, Sharma VK, Robinson T, et al. Rationale, design, and progress of the ENhanced Control of Hypertension And Thrombolysis strokE stuDy (ENCHANTED) trial: an international multicenter $2 \times 2$ quasi-factorial randomized controlled trial of low- vs. standard-dose rt-PA and early intensive vs. guideline-recommended blood pressure lowering in patients with acute ischaemic stroke eligible for thrombolysis treatment. Int J Stroke. 2015:10:778-88

5. Department of Health. The Handbook to the NHS Constitution. London: Department of Health; 2010.

6. Rhyne R, Sussman AL, Fernald D, Weller N, Daniels E, Williams RL, et al. Reports of persistent change in the clinical encounter following research participation: a report from the Primary Care Multiethnic Network (PRIME Net). J Am Board Fam Med. 2011;24:496-502.

7. Ozdemir BA, Karthikesalingam A, Sinha S, Poloniecki JD, Hinchliffe RJ, Thompson MM, et al. Research activity and the association with mortality. PLoS One. 2015;10:e0118253.

8. Jonker L, Fisher SJ. NHS Trusts' clinical research activity and overall CQC performance - is there a correlation? Public Health. 2015:129:1491-5.

9. Bennett WO, Bird JH, Burrows SA, Counter PR, Reddy VM. Does academic output correlate with better mortality rates in NHS trusts in England? Public Health. 2012;126:S40-3.

10. Downing A, Morris E, Corrigan N, Sebag-Montefiore D, Finaln P, Thomas J, et al. High hospital research participation and improved colorectal cancer survival outcomes: a population-based study. Gut. 2017;66(1):89-96. https:// doi.org/10.1136/annrheumdis-2015-311308.

11. Majumdar SR, Roe M, Peterson E, Chen A, Gibler B, Armstrong P. Better outcomes for patients treated at hospitals that participated in clinical trials. Arch Intern Med. 2008;168:657-62.

12. Krzyzanowska MK, Kaplan R, Sullivan R. How may clinical research improve healthcare outcomes? Ann Oncol. 2011;22(Suppl 7):vii10-5. 
13. Hanney S, Boaz A, Soper B, Jones T. Engagement in Research: An Innovative Three-stage Review of the Benefits for Health-care Performance. Health Services Delivery Research. Southampton: NIHR Journals Library; 2013.

14. Harding K, Lynch L, Porter J, Taylor N. Organisational benefits of a strong research culture in a health service: a systematic review. Aust Health Rev. 2017:41(1):45-53. https://doi.org/10.1071/AH15180.

15. Clarke M, Loudon K. Effects on patients of their healthcare practitioner's or institution's participation in clinical trials: a systematic review. Trials. 2011;12:16.

16. Litchen CA, Marsden G, Pollitt A, Kiparoglou V, Channon KM, Sussex J. Does a biomedical research centre affect patient care in local hospitals? Health Res Policy Syst. 2017:15:2.

17. Bray BD, Campbell J, Cloud GC, Hoffman A, Tyrrell PJ, Wolfe CDA, et al. Bigger, faster. Associations between hospital thrombolysis volume and speed of thrombolysis administration in acute ischemic stroke. Stroke. 2013; 44:3129-35.

18. Stroke Sentinel National Audit Programme. https://www.strokeaudit.org/. Accessed 7 Dec 2018.

19. Wahlgren N, Ahmed N, Davalos S, Ford GA, Grond M, Hacke W, et al. Thrombolysis with alteplase for acute ischaemic stroke in the Safe Implementation of Thrombolysis in Stroke-Monitoring Study (SITS-MOST): an observational study. Lancet. 2007;369:275-82.

20. Quinn TJ, Dawson J, Walters MR, Lees KR. Reliability of the modified Rankin scale: a systematic review. Stroke. 2009;40:3393-5.

21. Foerch C, Ghandehari K, Xu G, Kaul S. Exploring gender distribution in patients with acute stroke: A multi-national approach. J Res Med Sci. 2013; 18:10-6.

22. The IST-3 Collaborative Group. The benefits and harms of intravenous thrombolysis with recombinant tissue plasminogen activator within $6 \mathrm{~h}$ of acute ischaemic stroke (the third international stroke trial [IST-3]): a randomised controlled trial. Lancet. 2012;379:2352-63.

Ready to submit your research? Choose BMC and benefit from:

- fast, convenient online submission

- thorough peer review by experienced researchers in your field

- rapid publication on acceptance

- support for research data, including large and complex data types

- gold Open Access which fosters wider collaboration and increased citations

- maximum visibility for your research: over $100 \mathrm{M}$ website views per year

At $\mathrm{BMC}$, research is always in progress.

Learn more biomedcentral.com/submissions 